\title{
Development of CAM system for 3D surface machining with $\mathrm{CNC}$ lathe
}

\author{
Keigo TAKASUGI*, Yoshitaka MORIMOTO*, Katsuhiro NAKAGAKI** and Yoshiyuki KANEKO** \\ * Kanazawa Institute of Technology, \\ 7-1 Ogigaoka, Nonoichi-shi, Ishikawa, 921-8501, Japan \\ E-mail: ktaka@neptune.kanazawa-it.ac.jp \\ ** Technical Division, Takamatsu Machinery CO., LTD \\ 1-8 Asahigaoka, Hakusan-shi, Ishikawa, 924-8558, Japan
}

Received 28 February 2014

\begin{abstract}
We have developed a new machining method which realizes turning of non-axisymmetric curved surfaces with a rotation axis (called NACS-Turning hereafter). NACS-Turning is a CNC turning method with 3-axis synchronous which is composed of a turning axis and two translation axes. This method forms a profile of a non-axisymmetric curved surface by adopting a liner motor in $\mathrm{X}$ axis and by synchronizing the $\mathrm{X}$ axis with the rotation axis at high speed. We confirmed dramatic improvement of productivity by using this machining method. However, at present, the generation of tool paths for shape forming using NACS-Turning are obtained with APIs associated with a commercial 3D-CAD program and geometric calculations. Therefore, we have developed a new CAM application for NACS-Turning in this paper. Since this machining method is three-axis simultaneous control, even though composition of the axis is the same as a general two-axis turning lathe, tool paths have to be generated in three dimensional space. Therefore, applying for a tool generation method of a machining center is required. Specifically, machining points are generated by calculating intersection lines on free surfaces as machining surfaces are calculated, and coordinates of tool center points are calculated by using the inverse offset method. In this paper, first, outline of NACS-Turning is mentioned. Next, the detail of the developed CAM system which is limited to a rotary tool. Finally, we report effectiveness by cutting experimentation.
\end{abstract}

Key words: CAM, 3D machining, Turning, Non-axisymmetric surface, Rotary tool

\section{Introduction}

At present, non-circular shapes such as cam profiles or pistons for internal combustion engines are machined by cutting or grinding using a dedicated machining tool (Yoneda, et. al., 1990) (Mi-Ching and Yaw-Hwei, 1999). Since there has been a need to machine various mechanical parts that are composed of non-axisymmetric 3D surfaces with a rotation axis, not only dedicated machining tools but also general multi-purpose machine tools have commonly been used. However, in this case, non-circular shapes are machined by milling. This means that a turning spindle is used for the index axis. Therefore, if we need to obtain a specific surface roughness, the machining time will definitely increase.

In order to solve this problem, non-axisymmetric curved surface turning (called "NACS-Turning" hereafter) has been developed in our study (Morimoto, et. al., 2012a, 2012b). In NACS-Turning, the $X$ axis is driven with a high-speed linear motor while the tool is constantly fed in the $Z$-axis direction. This enables non-circular machining to be synchronized with the rotation of the turning spindle. We have confirmed that the $X$ axis can be driven by maximum acceleration of $4.5 \mathrm{G}$ and can follow a spindle revolution of $750 \mathrm{rpm}$.

Thus, NACS-Turning demonstrates a sufficient ability of the hardware to create curved surfaces by turning. However, there are no CAM applications that support this special machining method. At this moment, tool paths are computed using geometric calculations and APIs associated with a commercial 3D CAD program, which lacks versatility. Therefore, this paper proposes a new versatile tool path generation method for NACS-Turning. This 


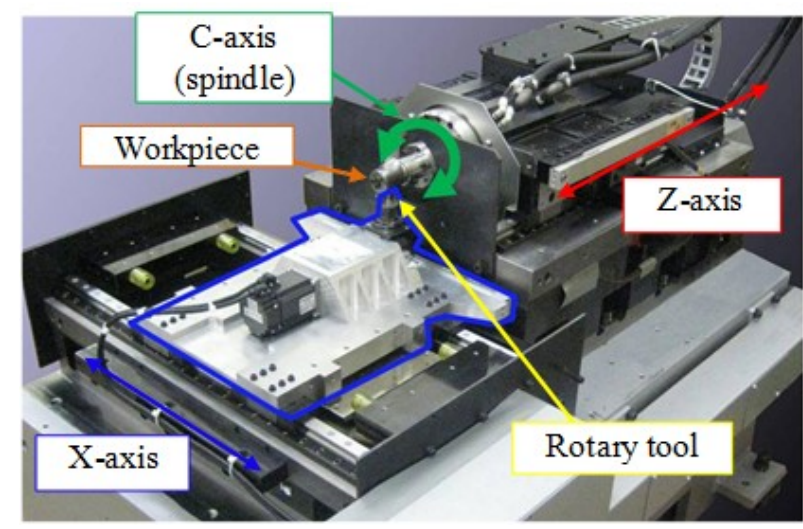

Fig. 1 CNC lathe for NACS-Turning

Table 1 Specifications of CNC NACS-Turning lathe

\begin{tabular}{|l|l|l|l|}
\hline Items & Specification & Value & Eng. unit \\
\hline Head & Max. spindle speed & 10000 & $\mathrm{rpm}$ \\
stock & Power of main motor & $1.0 / 2.64$ & $\mathrm{~kW}$ \\
& Stroke in Z direction & 200 & $\mathrm{~mm}$ \\
& Max. acceleration in Z dir. & 12.1 & $\mathrm{~m} / \mathrm{s} 2$ \\
\hline \multirow{2}{*}{ Carriage } & Stroke in X direction & 90 & $\mathrm{~mm}$ \\
& Max. acceleration in X dir. & 98.0 & $\mathrm{~m} / \mathrm{s}^{2}$ \\
& Mass & 26.4 & $\mathrm{~kg}$ \\
\hline & Total size & $720 \times 498 \times 1300$ & $\mathrm{~mm}$ \\
\hline
\end{tabular}

machining method requires generating tool paths in three dimensional space given that the two translation axes and the turning spindle are controlled by synchronizing their motions unlike in general turning.

In this paper, the CAM application for this special method is reported, and its effectiveness is confirmed.

\section{System configuration}

\subsection{CNC lathe for NACS-Turning}

Figure 1 shows the CNC lathe developed for NACS-Turning in this study. As mentioned above, it is composed of a turning axis ( $C$ axis) and two translation axes ( $X$ and $Z$ axes). The $C$ axis is on the $Z$ axis, and the carriage and tool are arranged on the independent $X$ axis. The tool is mounted in the vertical upward direction considering its selectivity and stiffness.

Table 1 shows the main specifications of the CNC lathe for NACS-Turning. The maximum speed of the spindle is $10000 \mathrm{rpm}$, the maximum acceleration in the $X$-axis direction is $10 \mathrm{G}$, and the stroke in the $X$ direction is $90 \mathrm{~mm}$. Furthermore, the positioning in the $X$ direction is controlled by a closed-loop with a linear scale resolution of $10 \mathrm{~nm}$.

\subsection{Tool layout}

Since this study aims at machining the high hardness materials for non-axisymmetric workpieces, a driven type rotary tool is targeted for CAM development. The tool is mounted in a unique position that is offset in the $Y$ direction with $l$ from the center of the $C$ axis. This means that a shank of the rotary tool can avoid the interference to a workpiece. The feed rate $f$ of the $Z$ axis is constant to the $-Z$ direction. Where, the diameter $D_{i}$ of the workpiece changes continuously in each cutter points: the $X$ coordinate values of the cutter points on the rotary tool changes continuously through machining process.

\subsection{CAM kernel}

This study develops a CAM application for NACS-Turning by using open CAM kernel "Kodatuno" (Takasugi, et. 

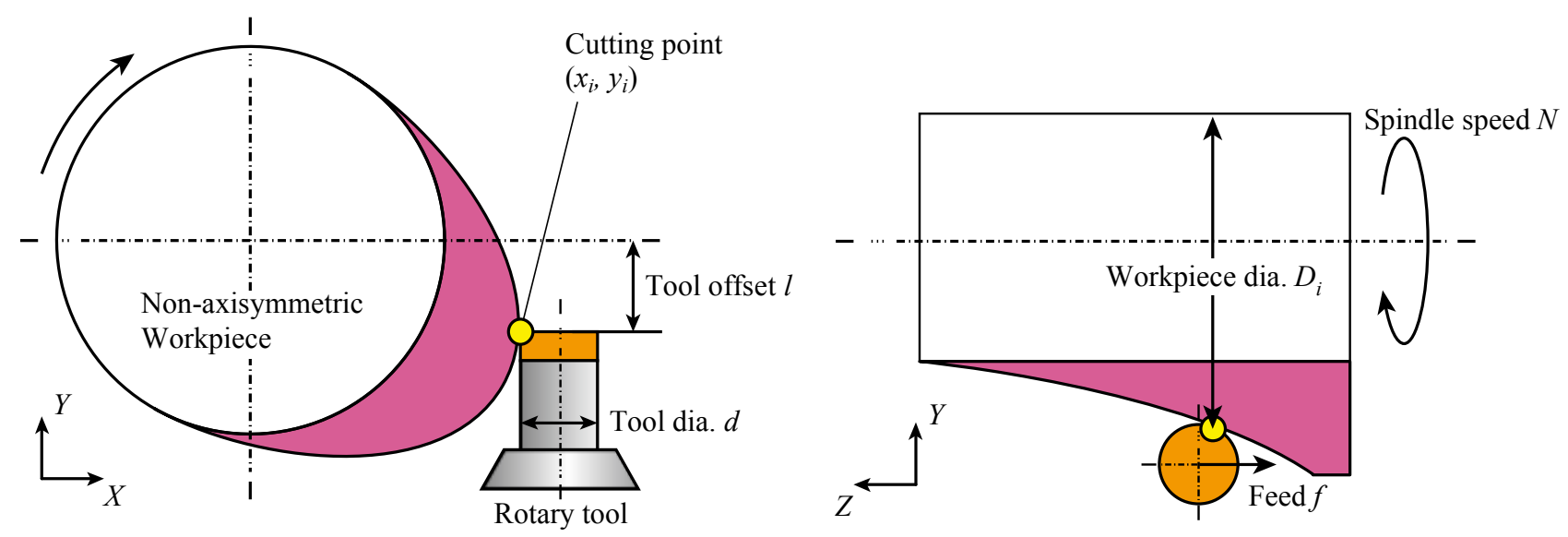

Fig. 2 Tool layout

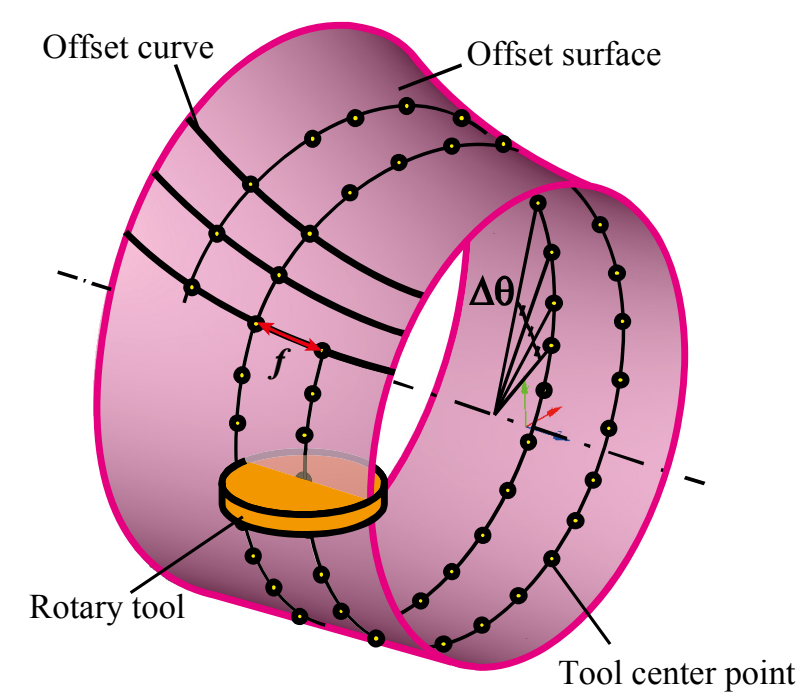

Fig. 3 Outline of cutter path generation

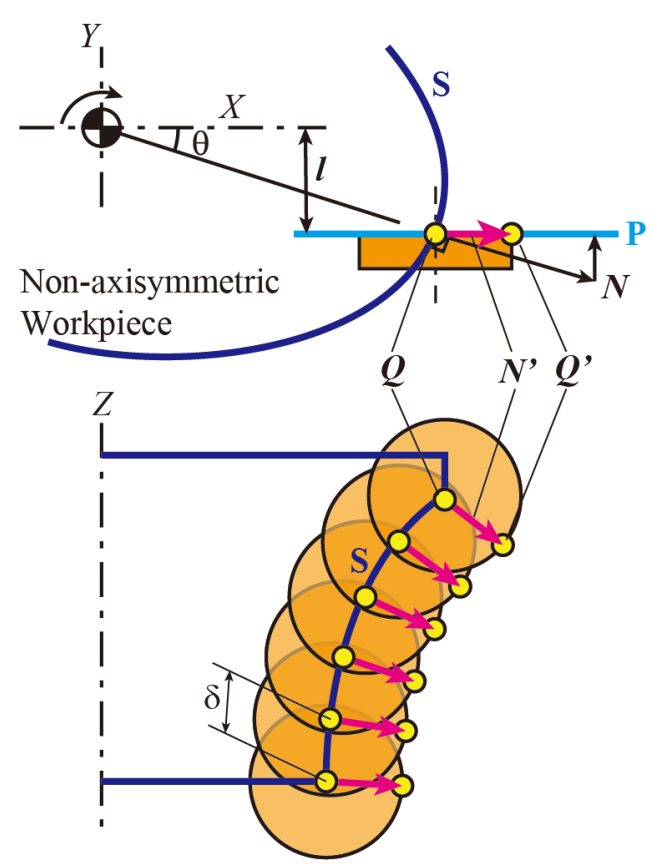

Fig. 4 Tool center trajectory with inverse offset method

al., 2011) instead of APIs for a general 3D CAD. Kodatuno is a surface kernel with open source, which has been developed by the first author in Kanazawa University. All input 3D models are converted to NURBS (Non Uniformed Rational B-Spline) expression and preserved. By this configuration, it will enable to derive the uniform geometric calculation across the entire CAM application. Moreover, since we can touch directly to NURBS expression, redundancy and error can be reduced to the minimum compared with the general CAM application development with AIPs in the commercial 3D CAD.

\section{Cutter path generation method}

Typically, in a process of a tool path generation with a ball end mill, ball center point that is offset at tool radius is adopted for a tool path. In this case, tool path generation methods are divided into two types as follows.

(1) Generating envelope surfaces with tool radius offset in advance, and generating a tool path on the offset surface.

(2) Generating points on original surfaces, and offsetting these points to normal directions with tool radius.

The method (1) has a disadvantage that it is difficult to keep enough accuracy because the envelope surfaces are interpolated surfaces with approximation operations. However, Points for stool path can be generated at regular pitch easily. On the other hand, while the method (2) does not require approximate calculation for tool center point 
generation, there is a disadvantage that offsets points will be irregular pitch.

In this machining method, since the rotation of the $\mathrm{C}$ axis and feed motion have to synchronize, it is prerequisite that the points for the tool path are regular pitch in the $Z$ direction. Furthermore, as shown in Fig. 3, if the number of control points per rotation is fixed, since angle pitch between each control point $\Delta \theta$ is constant $(2 \pi / n)$, interpolated curves connected to the $Z$ direction with points at each rotation angles only have to be generated: it does not require consideration of interpolating surfaces. Therefore, this study adopts the method (1).

A tool path generation for NACS-Turning is generated with three processes as described the next sections.

\subsection{Generation of tool center points}

As shown in Fig. 4, the inverse offset method (Kondo, et. al., 1988) is employed for generation of tool center point trajectories. Concrete flow is divided into following five processes.

(1) At a rotation angle $\theta$, intersection points $\boldsymbol{Q}$ between a plane $\mathbf{P}$ which corresponds to an upper plane on the rotary tool and the workpiece surface S (Higashi, et. al., 1990). Where, the calculation pitch $\delta$ of $\boldsymbol{Q}$ effects an approximate error to the next step, generation of NURBS interpolating curves.

(2) Normal vectors $\boldsymbol{N}$ in each $\boldsymbol{Q}$ on the surface $\mathbf{S}$ are calculated.

(3) $\boldsymbol{N}$ is projected to plane $\mathbf{P}$.

(4) Tool center point trajectories $\boldsymbol{Q}^{\prime}$ at the rotation angle $\theta$ are obtained by normalizing projected vectors considering tool radius $d / 2$.

(5) Processed from rotation axis 0 to $2 \pi$ in every angle pitch $\Delta \theta$, every tool center point trajectories are calculated.

\subsection{Generation of NURBS interpolation curves}

Offset NURBS interpolated curves in each $\theta$ are generated for trajectories from calculated tool center points. Since this machining method does not require interpolated surface, approximation error to the rotation direction depends on only angle pitch $\Delta \theta$. On the other hand, the approximation error to the $Z$ direction depends on interpolation points as mentioned above section. Envelope arc curves are added at the edges. The arc curves are expressed by NURBS.

\subsection{Generation of cutter location data}

Conclusive coordinates of machining points are calculated by obtained each offset NURBS interpolated curves and plane $\mathbf{R}$ which has $z$ coordinate value of cutter location and normal vector to the $Z$ direction. The $\mathrm{z}$ value is defined as follows:

$$
z=f\left(i+\frac{j}{n}\right) \quad \text { where, }\left\{\begin{array}{c}
0 \leq i<L / f \\
0 \leq j<n
\end{array}\right.
$$

where $f$ is feed rate per spindle rotation, $n$ is the number of divisions per rotation (the number of offset NURBS interpolation curves), and $L$ is cutting length of feed direction. Intersection points between $\boldsymbol{C}$ and $\boldsymbol{R}$ is calculated as follows:

$$
\boldsymbol{R}_{n} \cdot(\boldsymbol{C}(t)-\boldsymbol{p})=0
$$

where $\boldsymbol{R}_{\boldsymbol{n}}$ is normal vectors of $\mathbf{R}$, and $\boldsymbol{p}$ is a point on the $\mathbf{R}$. If NURBS degree number of the formula (2) is lower than four degrees, the formula can solve algebraically. However in this report, convergent calculation by Newton method is implemented in whole cases.

\section{Verification of developed CAM system}

In order to verify the effectiveness of developed CAM system for NACS-Turning, generation of CL data and accuracy of the tool paths and machining are conducted.

\subsection{Verification of proposed CL data generation method}




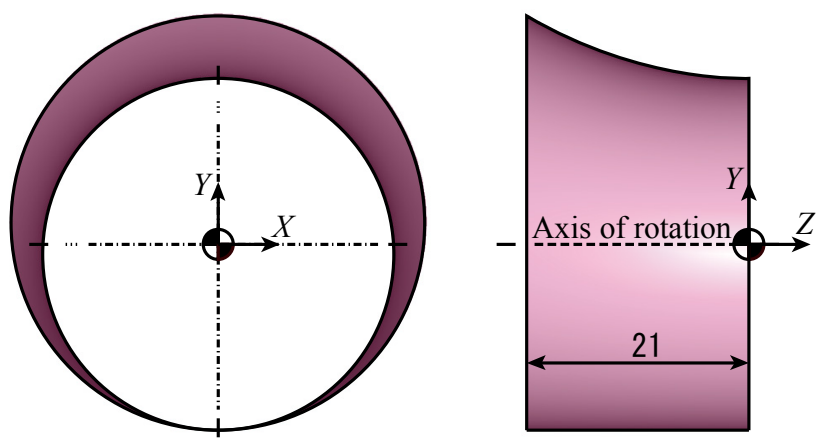

Table 2 Cutting conditions

Fig. 5 Workpiece for verification

\begin{tabular}{|c|c|c|}
\hline & Value & Eng. unit \\
\hline Spindle speed & 375 & $\min ^{-1}$ \\
\hline Feed & 0.2 & $\mathrm{~mm} / \mathrm{rev}$ \\
\hline Depth of cut & 0.2 & $\mathrm{~mm}$ \\
\hline Tool offset & 7.5 & $\mathrm{~mm}$ \\
\hline Division per revolution & \multicolumn{2}{|c|}{720} \\
\hline \multirow{3}{*}{$\begin{array}{l}\text { Calculation pitch of } \\
\text { interpolation points } \delta\end{array}$} & 1.0 & \multirow{3}{*}{$\mathrm{mm}$} \\
\hline & 0.5 & \\
\hline & 0.2 & \\
\hline Workpiece & \multicolumn{2}{|c|}{ SKD11 (HRC60) } \\
\hline
\end{tabular}

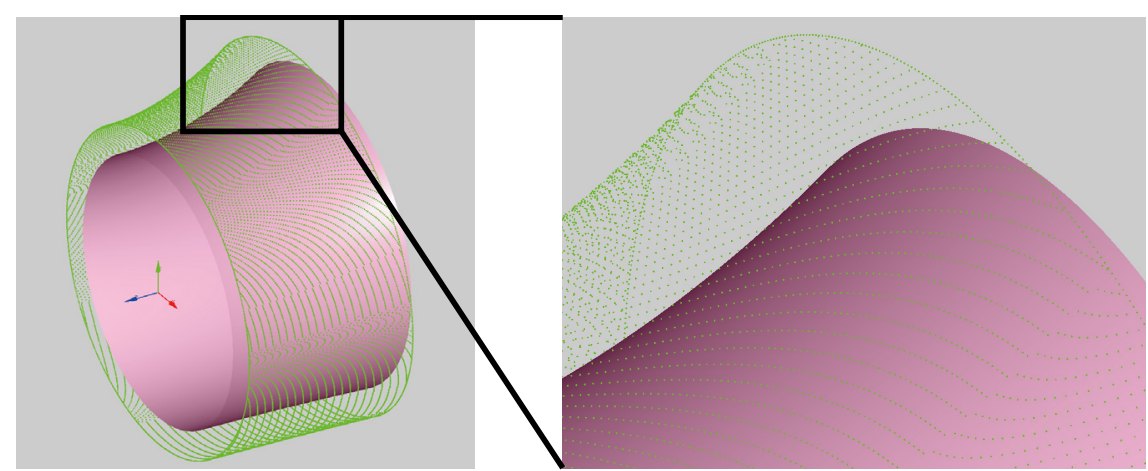

Fig. 6 Result of tool center point

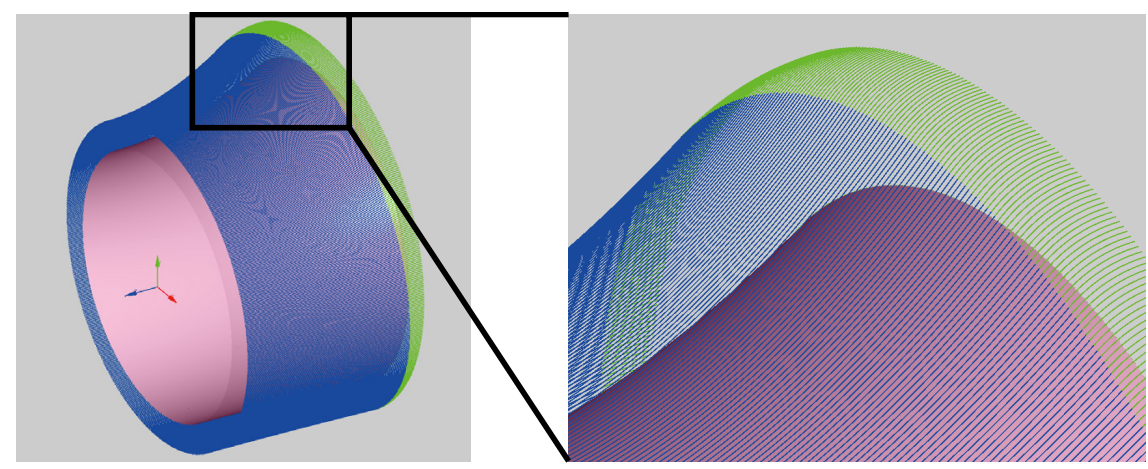

Fig. 7 Result of NURBS interpolation curve

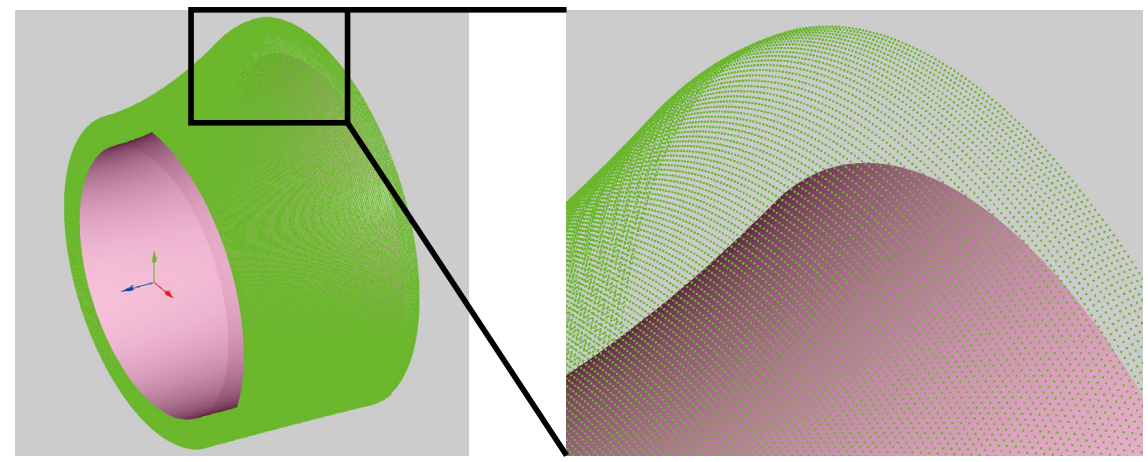

Fig. 8 Result of Cutter location generation

Figure 5 shows a workpiece used for verification. Cutting length is $21 \mathrm{~mm}$. Diameter of workpiece is enlarged incrementally from the edge face to $-Z$ direction. A center of the circle changes its position depending on the that of the rotation axis to $+Y$ direction. Necessary cutting conditions for generation of CL data are shown in table 2 . The number of divisions per rotation is 720 , and the rotational angle pitch $\Delta \theta$ of cutting points is $0.5^{\circ}$. From formula (1), the distance between cutting points to the $Z$ direction is set to $2.78 \times 10^{-4} \mathrm{~mm}$. Three types of calculation pitch $\delta$ of intersection points are verified to examine the effect of $\delta$. In this verification, a workstation (OS: Windows7 64bit CPU: 
Takasugi, Morimoto, Nakagaki and Kaneko,

Journal of Advanced Mechanical Design, Systems, and Manufact, Vol.8, No.4 (2014)

Table 3 Comparison of processing time in each calculation pitch of intersection points

\begin{tabular}{|l|c|c|c|c|c|}
\hline \multicolumn{2}{|c|}{} & \multicolumn{4}{|c|}{ Processing time [s] } \\
\cline { 2 - 6 } \multicolumn{2}{|c|}{} & $\begin{array}{l}\text { Calculation of } \\
\text { tool center points }\end{array}$ & $\begin{array}{l}\text { Interpolation of } \\
\text { NURBS curves }\end{array}$ & $\begin{array}{l}\text { Generation of } \\
\text { cutter location data }\end{array}$ & Total \\
\hline \multirow{3}{*}{$\begin{array}{l}\text { Calculation } \\
\text { pitch } \delta\end{array}$} & $1.0 \mathrm{~mm}$ & 88.4 & 0.21 & 20.7 & 109.3 \\
\cline { 2 - 6 } & $0.5 \mathrm{~mm}$ & 162.0 & 0.66 & 41.7 & 204.4 \\
\cline { 2 - 6 } & $0.2 \mathrm{~mm}$ & 387.8 & 4.2 & 103.2 & 495.2 \\
\hline
\end{tabular}

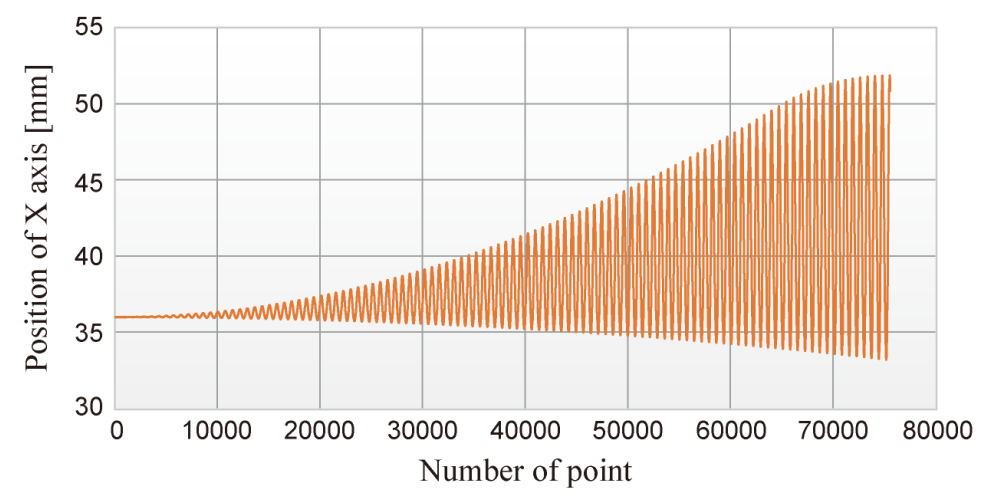

Fig. 9 Change of position of $\mathrm{X}$ axis $\left(\delta_{1.0}\right)$

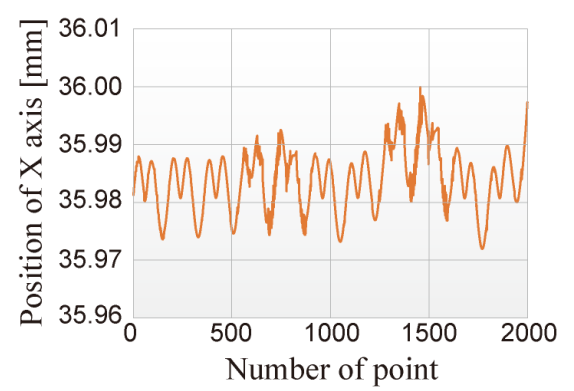

(a) In the case of $\delta_{1.0}$

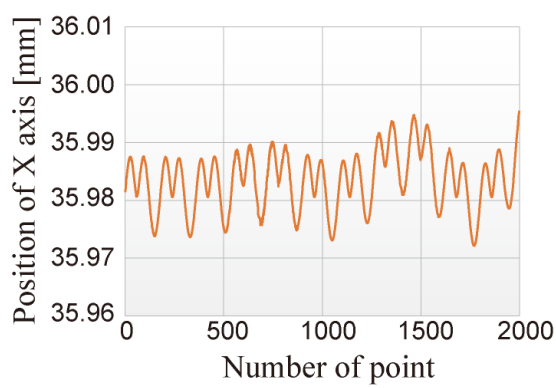

(b) In the case of $\delta_{0.5}$

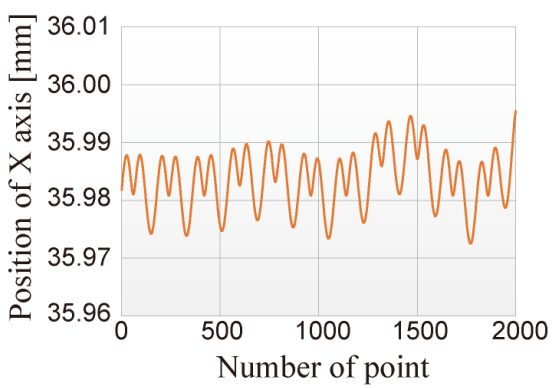

(c) In the case of $\delta_{0.2}$

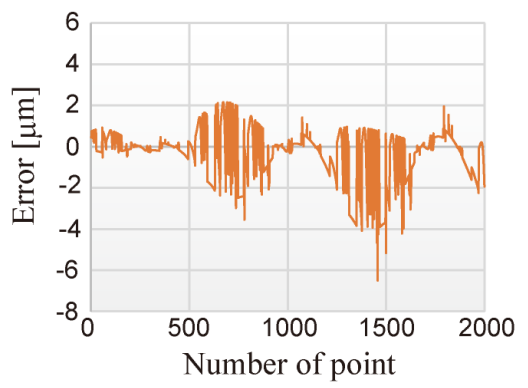

(d) Error distribution $\left(\delta_{1.0}-\delta_{0.2}\right)$

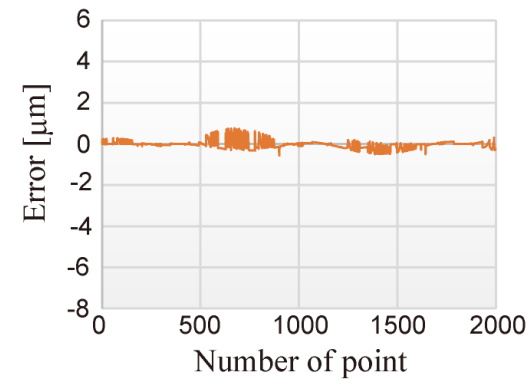

(e) Error distribution $\left(\delta_{0.5}-\delta_{0.2}\right)$

Fig. 10 Difference of error between conditions of calculation pitch $\delta$

Table 4 Maximum value of difference between each $\delta$

\begin{tabular}{|l|c|c|c|}
\hline & $\delta_{1.0}-\delta_{0.2}$ & $\delta_{0.5}-\delta_{0.2}$ & $\delta_{1.0}-\delta_{0.5}$ \\
\hline Error $[\mu \mathrm{m}]$ & 8.4 & 1.1 & 8.4 \\
\hline
\end{tabular}

Xeon3.60GHz, 8 Cores, Memory: 16GB) is employed.

Figure 6 shows a generated tool center point's trajectories when $\delta$ equals $1.0 \mathrm{~mm}$. Next, Fig. 7 shows a generation result of offset NURBS interpolated curves from tool center point trajectories. Blue lines in the figure show the offset curves on machining surface on the workpiece, and green lines show the envelope curves with arc on the edge part. Finally Fig. 8 shows generated cutting points from offset NURBS interpolated curves. Spiral cutting points with feed of $0.2 \mathrm{~mm}$ from the edge surface $(Z=0)$ to the $Z$ direction are generated. Also in the connection of envelope arcs on the edges, it shows that cutting points are generated without any irregular pitch. In the operation, the calculation time in 

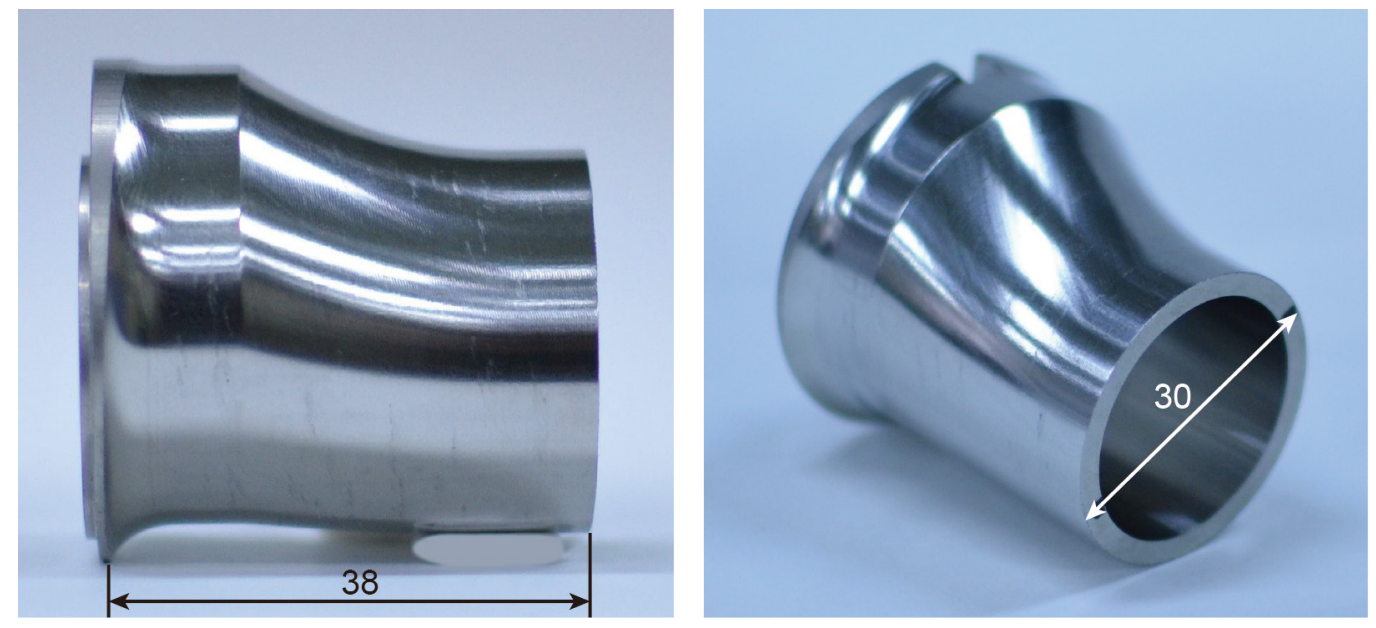

Fig. 11 Machining result

each $\delta$ is compared. The results are shown in table 3 . Each process is calculated by parallel processing with 8 cores using OpenMP. According to the results, as $\delta$ becomes lower, the calculation time increases in all three calculation processes. Also, it is determined that the total calculation time is approximately inversely proportional to each $\delta$.

\subsection{Effect of calculation pitch}

Comparison of CL data between calculation pitch $\delta=1.0 \mathrm{~mm}, 0.5 \mathrm{~mm}$ and $0.2 \mathrm{~mm}$ is conducted. Where, an index of $\delta$, e.g. $\delta_{1.0}$, means the calculation pitch hereafter. Fig. 9 shows the travel distance of the $\mathrm{X}$ axis of CL data at $\delta_{1.0}$. There are no discontinuous area through machining, and it appears that the $\mathrm{X}$ axis can be driven smoothly. However, since calculated points approach curves on the machining surface $\boldsymbol{S}$, it is assumed that $\delta_{0.2}$ has few differences between the real shapes compared with $\delta_{1.0}$. In fact, as shown in Fig. 10, errors can be confirmed in each $\delta$ in closeup of a part. Especially at $\delta_{1.0}$, an error like noise in the order of $8 \mu \mathrm{m}$ relative to $\delta_{0.2}$ is confirmed as shown in Fig. 10 (a) and (d). However, at $\delta_{0.5}$, the error is reduced compared with $\delta_{1.0}$ as shown in this Fig. (b) and (e). Table 4 shows difference of the tool path between each $\delta$. From these results, $\delta_{1.0}$ has a margin of error of $8.4 \mu \mathrm{m}$ from $\delta_{0.2}$. However, since the difference between $\delta_{0.5}$ and $\delta_{0.2}$ is only $1.1 \mu \mathrm{m}$, it is conclude that the error for the calculation pitch converges exponentially.

\subsection{Verification of Machining}

We verified effectiveness of the generated tool path by using $\delta_{1.0}$. Machining conditions are shown in table 2 . Figure 11 shows the result of machining of a sample workpiece. The machined surface is sufficiently smooth at even $\delta_{1.0}$. The profile error on the tool path at $\delta_{1.0}$ has about $8 \mu \mathrm{m}$, however since all the machined surface is free surfaces, it is difficult to measure the actual machining error, which is our future work. Also, we confirmed the noise on the tool path about the amplitude of $2 \mu \mathrm{m}$. In fact, the rapid acceleration change of the $\mathrm{X}$ axis is smoothed by a control lag. Therefore, the error is not transferred directly to the machined surface (Morimoto, et. al., 2013).

\section{Conclusion}

In this paper, versatile a tool path generation method for NACS-Turning which enables to machine non-axisymmetric curved surfaces has developed, and we obtained the following results.

1. A CAM application for NACS-Turing was developed by using open CAM kernel "Kodatuno", and the availability was introduced.

2. The generation of tool center points with the pitch of $1 \mathrm{~mm}$ in intersection points showed enough accuracy for the tool path. 
Takasugi, Morimoto, Nakagaki and Kaneko,

Journal of Advanced Mechanical Design, Systems, and Manufact, Vol.8, No.4 (2014)

\section{References}

Higashi, M., Mori, T. and Hosaka. M., High-Quality Intersection Calculation of Surfaces by Geometrical Tracing of Intersections, Journal of Japan Society of Precision Engineering, Vol. 56, No. 1 (1990), pp. $92-97$ (in Japanese).

Kondo, T., Kishinami, T. and Saito, K., Machining System based on Inverse Offset Method, Journal of Japan Society of Precision Engineering, Vol. 54, No. 5 (1988), pp. 971-976 (in Japanese).

Mi-Ching, T. and Yaw-Hwei, L., Tracking Control of a Software Cam Machining System, Transactions of the ASME Journal of dynamic systems measurement and control, Vol. 121, No. 4 (1999), pp. 729-735.

Morimoto, Y., Emoto, S., Moriyama, T. and Kato, H., Creation of Curved Surface by Lathe Turning, Proceedings of CIRP, Vol. 1 (2012a), pp. 114-119.

Morimoto, Y., Emoto, S., Moriyama, T., Kato, H., Nakagaki, K. and Kaneko, Y., Realization of 3D Surface Machining by Turning (Proposal of Turning Method with Rotary Tool), Transactions of Japan Society of Mechanical Engineering Series C, Vol. 79, No. 804 (2013), pp. 2932-2951 (in Japanese).

Morimoto, Y., Moriyama, T. and Emoto, S., Development of Linear-Motor-Driven NC Table for High-Speed Machining of 3D Surface by Lathe Turning, Proceedings of CIRP, Vol. 1 (2012b), pp. 271-276.

Takasugi, K., Kumasaka, T. and Asakawa, N., Development of Platform-Independent Open CAM Kernel, Proceedings of LEM21, Vol. 1 (2011).

Yoneda, T., Suzuki, I. and Tsujiuchi, T., Study on NC Profile Grinding for Non-Circular Shapes, Journal of Japan Society of Precision Engineering, Vol. 56, No. 12 (1990), pp. 2297-2302 (in Japanese). 